\title{
Neuroethics and Nanoethics: Do We Risk Ethical Myopia?
}

\author{
Sheri Alpert
}

Received: 24 August 2007 / Accepted: 7 December 2007 / Published online: 7 February 2008

(C) Springer Science + Business Media B.V. 2008

\begin{abstract}
In recent years, two distinct trajectories of bioethical inquiry have emerged: neuroethics and nanoethics. The former deals with issues in neuroscience, whereas the latter deals with issues in nanoscience and nanotechnology. In both cases, the ethical inquiries have coalesced in response to rapidly increasing scientific and engineering developments in each field. Both also present major issues for contemplation in bioethics. However, the questions are (1) how different are the ethical issues raised, and (2) is it beneficial for neuroethics and nanoethics inquiries to proceed on oftendivergent trajectories by ethicists who otherwise might never interact? If, for example, ethical inquiry occurs only within the disciplinary confines of their predominant area(s) of science (which now seems to be the case) or by overlooking prior discussions in other scientific realms (like genetics), then the opportunity for a richer, more comprehensive discourse may be lost. I argue that this (1) is a disservice to bioethics, (2) is antithetical to some of the aims of bioethical inquiry, and (3) encourages the reductionism bioethicists' claim that is counterproductive.
\end{abstract}

\section{S. Alpert $(\bowtie)$}

Dalhousie University,

Halifax, Nova Scotia, Canada

e-mail: sheria@well.com
Keywords Neuroethics · Nanoethics · Genethics · Nanotechnology $\cdot$ Neuroscience .

Intra-disciplinary ethics

In recent years, two distinct trajectories of bioethical inquiry have emerged: neuroethics and nanoethics. The former deals with issues in neuroscience (the study of the brain and the nervous system) while the latter deals with issues in nanoscience and nanotechnology (an applied science focused on the design, synthesis, characterization, and application of materials and devices on a sub-atomic scale). In both cases, the ethical inquiries have coalesced in response to rapidly increasing scientific and engineering developments in each field. Both also present major issues for contemplation in bioethics. Moreover, the ethical issues each raises have been pursued largely independently of one another, which suggests that these issues in each case are somehow categorically unique to each context. This independent consideration could be justified only if, in fact, this were the case, and that nothing would be gained by ethicists interacting with those addressing ethical issues from each context. This article attempts to address the validity of this approach by examining what is meant by "neuroethics" and "nanoethics," what sorts of applications in each of the principal scientific domains raise ethical concerns, what is driving the subdivision of these ethical inquiries, and the consequences of separating the inquiries. 


\section{What is "Neuroethics" and Why the Recent Interest?}

Very generally speaking, neuroethics is the study of ethical issues associated with the brain, and/or neuroscience and neurotechnology. However, this definition can be further refined. Walter Glannon defines neuroethics as a

branch of bioethics concerned with the ethical issues arising from different measures of and interventions in the brain or central nervous system. It lies at the intersection of the empirical brain sciences, normative ethics, philosophy of mind, law, and the social sciences ([18], p. 4).

Likewise, Michael Gazzaniga describes neuroethics in a richer way, as being "more than just bioethics for the brain" - for him it is

the examination of how we want to deal with the social issues of disease, normality, mortality, lifestyle, and the philosophy of living informed by our understanding of underlying brain mechanisms (emphasis in the original; [16], xv).

Gazzaniga's definition is a broad one that takes into account our knowledge of brain function in identifying and addressing the ethical and social issues arising from our study and potential manipulation thereof.

There is some debate about when neuroethics arose as a separate area of ethical reflection. Certainly, ethical questions have arisen from neuroscience, in both clinical and research contexts, over the course of many years. However, Illes ties the launch of neuroethics as a discipline to a Dana Foundation conference held in May 2002 ([19], 1739). The conference was one of the first that brought together neuroscientists, lawyers, bio- and medical ethicists, and policy-makers to discuss various aspects and implications of advances in neuroscience.

Since that time, an ever increasing number of articles has been written about many different topics that fall under the general topic of "neuroethics." As neuroscientists make more progress in their understanding of how the brain functions, no doubt the ethical implications of that understanding will continue to develop, as will the array of issues and applications for which ethical implications are identified.

\section{Neuroethics: Some of the Issues}

There are many different categories of issues that arise from our increasing understanding of brain mechanisms. Among the most often discussed issues is the use of functional neuroimaging. For instance, fMRI (functional magnetic resonance imaging) has been used in a number of research settings to study "the neural correlates of a range of physical and mental health conditions, behaviors, preferences, and characteristics" ([45], p. 1). fMRI has been used in studying everything from consumers' preferences for one brand of soda over another (e.g., [27]), to altruism (e.g., [43]) and social cooperation (e.g., [11, 13]), to the detection of the intention to lie (e.g., [1]). In none of these cases is the use of fMRI currently scientifically robust enough for ethical use outside a research setting. Another set of issues identified within neuroethics concerns neuroenhancement. These issues generally involve psychopharmaceuticals; i.e., the use of anti-depressants particularly in non-depressed people, the use of stimulants like Ritalin or modafinil in people who suffer from neither attention deficit disorder nor narcolepsy, and the use of beta blockers (e.g., propranalol) for post-traumatic stress disorder sufferers, to interfere with the brain's ability to imprint the emotional valence associated with the memories of traumatic events.

\section{What is "Nanoethics" and Why the Recent Interest?}

Nanoethics addresses the ethical, social, and policy issues associated with developments and applications in nanoscience and nanotechnology. Like so many other areas of science and ethics, there are no consensus definitions for "nanoscience" and "nanotechnology." In very general terms, however, nanoscience "is the study of phenomena and manipulation of materials at the atomic, molecular, and macromolecular scales, where properties differ significantly from those at a larger scale" (emphasis added) [28]. According to the National Nanotechnology Initiative, nanotechnology is

the understanding and control of matter at dimensions of roughly 1 to 100 nanometers, where unique phenomena enable novel applica- 
tions. Encompassing nanoscale science, engineering and technology, nanotechnology involves imaging, measuring, modeling, and manipulating matter at this length scale. At the nanoscale, the physical, chemical, and biological properties of materials differ in fundamental and valuable ways from the properties of individual atoms and molecules or bulk matter. Nanotechnology R\&D is directed toward understanding and creating improved materials, devices, and systems that could use these new properties ([34], p. 4).

To get a brief appreciation of the size of things in the nanoscale, consider the following. A "nanometer" (nm) equals one billionth of a meter. It takes ten atoms of hydrogen side-by-side to equal one nanometer. A DNA molecule is about 2.5 -nm wide. A red blood cell is vast in comparison: about 5,000-8,000 $\mathrm{nm}$ in diameter. One human hair is approximately $80,000-\mathrm{nm}$ thick, and the head of a pin is approximately 2 million nanometers wide. Everything on the nano-scale is invisible to the unaided eye and even to all but the most powerful microscopes, such as the scanning tunneling microscope and the atomic force microscope.

Nanotechnology encompasses many different types of materials and applications. However, it does not refer to any specific materials or applications. "Nano" (whether science or technology) refers only to the scale of the object. This contrasts with "neuroscience" which refers specifically to the brain and nervous system, or "bioscience" which relates to biological systems.

Nanotechnology is a relatively young field of endeavor, getting its conceptual start with a speech in December 1959 by Richard Feynman. In that speech, called "There's Plenty of Room at the Bottom," Feynman discussed "the problem of manipulating and controlling things on a small scale" [14]. Indeed, Feynman offered a prize of $\$ 1,000$ during the speech to the first person who could take the text on a page of a book and shrink it to $1 / 25,000$ of its original size, so that it could be read with an electron microscope. The prize was not awarded until 1985. Progress in nanoscience and nanotechnology has accelerated markedly since that time. For instance, there are already 580 consumer products using nanotechnology [30] having more than doubled in number in the previous 18 months. While use in consumer products is gaining ground, this is far from the only (or even, arguably, the most lucrative) context within which nanotechnologies have potential application. There are also commercial applications, which are projected to be worth around \$2.6 trillion by 2014 [26].

The remainder of this discussion will consider nanoscience and nanotechnology within the medical research and clinical contexts. This facilitates comparisons and contrasts in areas relevant to bioethics. Within the specific area of nanomedicine, several categories of application are foreseen, such as diagnostics, drug delivery, and tissue engineering. As described in a recent report,

[i]f scientists domesticate atoms and molecules, they could harness them for a wide range of medical purposes. For one thing, they could create novel nanostructures that serve as new kinds of drugs for treating common conditions such as cancer, Parkinson's and cardiovascular disease. They could also engineer nanomaterials for use as artificial tissues that would replace diseased kidneys and livers, and even repair nerve damage. Moreover, they could integrate nanodevices with the nervous system to create implants that restore vision and hearing and build prosthetic limbs that better serve the disabled. When you consider that one-third of all Americans are Baby Boomers - and that many others already suffer from degenerative disease - it is clear that advances in nanomedicine could help vast numbers of people maintain their health, their independence and their participation in society ([42], p. 29).

Clearly, there are many optimistic predictions about the positive impact of nanotechnology for applications within medicine. Indeed, scientists have already begun to make substantial progress with nanotechnology in the area of targeted drug delivery (e.g., [9, 10, 41]), although practical human applications are still several years away. And while these applications sound remarkable, they are not uncontroversial.

\section{Relationship Between Nanotechnology and Neuroscience}

There are several areas in which nanotechnology and neuroscience overlap. Some have pointed to the areas 
Fig. 1 Relationship between Nanotechnology and other sciences

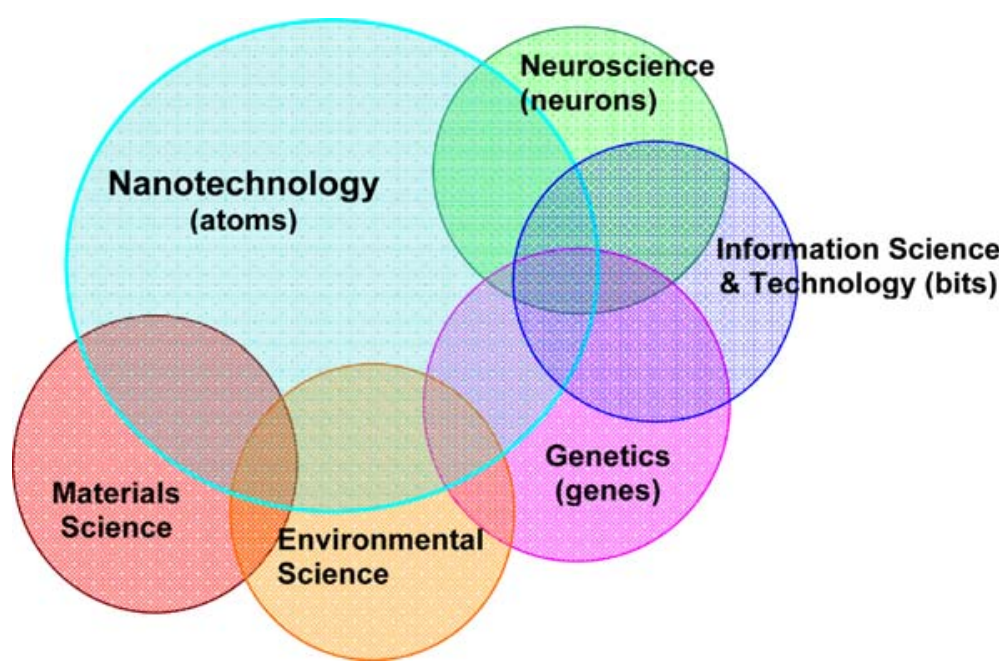

of overlap as reason to resist separating ethical inquiries pertaining to either of the two areas. Others, however, point to the areas where there is no overlap to stress each area's uniqueness, and why the ethical issues arising from each should be treated separately. In either case, it is important to understand the nature of the relationship between the two disciplines to intelligently discuss the ethical issues arising from each, and whether there is anything to be gained by separating the discussions.

Figure 1 depicts, in very rough terms, some of the overlaps between nanotechnology and other sciences. As you will notice, there are many different sciences that overlap with nanotechnology. The reason for that is simple: as stated above, nanotechnology refers only to scale, and not specifically to any scientific/ technological domain.

Also note that this diagram is vastly oversimplified. Its intent is to convey that nanotechnologies are applicable in many different sciences, any or all of which will engender ethical quandaries at some point. It is also worth noting that the areas of science, themselves, overlap with each other. ${ }^{1}$ Particularly note the overlaps between neuroscience, genetics, and information sciences. In combination with nanotechnology, these domains comprise the area of converging technologies dubbed by a US National Science Foundation report as "NBIC" technologies; i.e., nano-

\footnotetext{
${ }^{1}$ The diagram does not intend to imply that non-adjacent sciences depicted above do not necessarily overlap or have common applications. This is merely a limitation of the twodimensional space of the page.
}

bio-info-cogno [40]. NBIC technologies will integrate atoms, genes, data bits, and neurons. And just as the sciences intersect and overlap, so too will many of the ethical issues. This will be useful to bear in mind in the discussion that follows.

\section{Why the Move to Subdivide Ethics?}

There are many possible reasons why there have been separate ethical inquiries into issues that arise in neuroscience and nanotechnology. These can be reduced to two:

1. Arguably it is more effective to address the ethical issues in the specific contexts within which they occur. For instance, there are many types of ethical issues that arise within nanotechnology that will not arise within the neurosciences, e.g., issues of environmental impact, issues inherent to the nature of nanoscience, endeavors to create new types of materials and devices by manipulating individual atoms and molecules, and the potential positive and negative economic impact nanotechnologies are predicted to have. Similarly, there are issues within the neurosciences that will not be relevant for nanotechnology - for instance, the ways in which fMRI images are interpreted and used (practically and methodologically).

2. It can also be argued that what is driving the separation of nano- and neuroethics is the fact that funding agencies (e.g., US federal agencies) are 
providing significant dollars for the contemplation of these issues within the context of a specific science or technology, such as nanotechnology or genetics. Pursuing sub-divided ethics, then, is a matter of pragmatism. After all, it makes sense in the academic environment, which generally rewards grant acquisitions, for researchers who examine ethical issues to apply their skills to the questions that agencies are generously funding.

Certainly the more plausible, if slightly cynical, of these possible reasons for separating nano- and neuroethics is provided by the second explanation. Funding amounts for at least two areas of "subdivided" ethics support this claim: from genetics (genethics) and nanotechnology (nanoethics).

The mapping of the human genome became a formalized program in 1990 (although funding began in 1988), and ran through 2003. A total of $\$ 3.7$ billion was spent on all the activities associated with the Human Genome Project (HGP), split between the US Department of Energy (DOE) and the National Institutes of Health (NIH). During the project, DOE set aside $3 \%$ of its annual HGP budget and NIH set aside $5 \%$ of its budget to study the project's ethical, legal, and social issues (ELSI) [17]. This amounts to a total of approximately $\$ 166.6^{2}$ million dollars over 13 years to study ELSI issues - i.e., genethics was funded to the tune of nearly $\$ 13$ million/year for 13 years. ${ }^{3}$ Moreover, the 5\% funding for ELSI issues has continued from the National Human Genome Research Institute (NHGRI) since the HGP officially ended. For instance, in 2004, NIH and DOE provided funding to launch four "Centers of Excellence" for ELSI research, and will have spent nearly \$20 million over the five year funding period [31], or approximately $\$ 4$ million year. Total funding for NHGRI ELSI activities has totaled \$56 million from 20042006 [6]. Between 1990-2003, approximately 420 ELSI grants were awarded by the NHGRI, with around 130 from 2004-2007 [32].

\footnotetext{
${ }^{2}$ The ELSI budget numbers were calculated from total funding figures provided at: http://www.ornl.gov/sci/techresources/ Human_Genome/project/budget.shtml.

${ }^{3}$ According to a communication from the ELSI staff of NHGRI [6], the NIH funding for ELSI projects from 1990-2003 may have been closer to $\$ 121$ million, for a total of $\$ 150$ million (including DOE funding). Whichever figure is correct, the amount of money made available for HGP ELSI research has been and continues to be significant.
}

The other major science and technology project that is currently underway in the US is the National Nanotechnology Initiative (mentioned above). This effort includes 26 federal agencies, 13 of which have nanotechnology research and development budgets. The agencies comprising the NNI provide much of the funding that is made available through grants to the academic community. By far, the National Science Foundation (NSF) provides the greatest share of dollars for grants on nanotechnology's societal dimensions. ${ }^{4}$ Funding has been tracked for nanotechnology spending since 2001, and specifically for ethical, legal, and other societal issues (ELoSI), since 2005. Since Education initiatives are also included in the funding numbers for the ELoSI area, these numbers overstate how much is actually going to ELoSI research vis-a-vis nanotechnology. With that caveat stated, the numbers for nanotechnology ELoSI spending have been fairly constant for the last few years, while funding for the environmental, health, and safety issues has increased, particularly in the request for 2008 funding. Funding for education and ELoSI was \$33.3 million in 2005, \$34.1 million in 2006, \$38 million in 2007 (estimated; [33], p. 39]. This represents approximately $2.6 \%$ of the annual NNI budget. Table 1 presents these numbers, along with the total spending for the NII for each year.

So, clearly, for humanities and social science scholars (i.e., those most likely to be doing ethics research), the Human Genome Project and the National Nanotechnology Initiative have proven to be lucrative lines of inquiry. Again, I acknowledge that this is a somewhat cynical view, and that it is not possible to know how much of this research would have occurred were it not for the funding. Indeed, at universities that are considered "research institutions," it would be difficult if not impossible to get tenure or advance an academic career without successfully competing for grants and publishing articles or books. So, to the extent that an academic's research interests are flexible, these funding sources for ELSI/ELoSI research have been a boon.

In neuroethics, the pattern is a bit different. There are no national/international coordinated initiatives to

\footnotetext{
${ }^{4}$ Monies are provided under the broad category of "societal dimensions", which includes funding for the examination of environmental, health, and safety issues (which are tracked as a class of funding within the societal dimensions category), and for education and ethical, legal, and other societal issues.
} 
Table 1. Funding for Nanotechnology Education and ELoSI Initiatives \& Total NNI Funding

\begin{tabular}{lll}
\hline Year & Funding & NNI total \\
\hline 2005 & $\$ 33.3$ million & $\$ 1,200.0$ million \\
2006 & $\$ 34.1$ million & $\$ 1,351.2$ million \\
2007 & $\$ 38.0$ million (requested) & $\$ 1,392.1$ million \\
2008 & $\$ 38.9$ million (proposed) & $\$ 1,444.8$ million \\
\hline
\end{tabular}

map the "brainome," although there has been increased interest in issues of neuroscience and neurotechnologies in the last several years. For instance, in 2004, the NIH launched the "Blueprint for Neuroscience Research," a collaborative effort between 16 NIH Institutes, Centers or offices [3]. The Blueprint's aim is to

develop research tools, create research resources shared by the entire neuroscience community, train a new generation of cross-disciplinary neuroscientists, and importantly, to develop a cooperative framework for the institutes and centers to plan and implement their neuroscience research efforts (ibid. p. 10329).

The Blueprint activities are funded from the general appropriations the centers and institutes receive, but account for only a small proportion of the overall funding for neuroscience. Indeed, according to Baughman, et al., the total funding for neuroscience research at the National Institutes of Health is approximately $\$ 4.167$ billion. $^{5}$

Unlike the Human Genome Research Project and the NNI, however, there is not an explicit dedicated percentage of money in the US that funds research into the ethical, legal, and social implications of either neuroscience research, the application of neuroscience tools, or interventions in the brain and central nervous system. However, several of the NIH institutes that cover the neurosciences do participate in the smaller multi-institute grant programs that provide funding for ELSI type issues. For instance, one call for proposals, "Research On Ethical Issues In Human Subjects Research" (PA-07-277) includes as potential grantors $17 \mathrm{NIH}$ institutes or offices, including ten

\footnotetext{
${ }^{5}$ Note that this number was calculated by this author based on the numbers provided in the Baughman, et al. article (p. 10329).
}

institutes that are also members of the NIH Blueprint for Neuroscience Research.

The major concerted effort being made to fund initiatives specifically in neuroethics is occurring in Canada. In its 2007 strategic plan, the Institute of Neurosciences, Mental Health and Addiction (INMHA) within the Canadian Institute for Health Research (CIHR) states it "targeted neuroethics as a novel niche area for Canadian experts" ([22], p. 23). The plan makes clear that officials at CIHR-INMHA have made a concerted effort in the area of neuroethics:

CIHR-INMHA organized an expert workshop on the topic in 2002 in Toronto and two cycles of RFAs [Requests for Proposals] were launched, with one team funded in each competition. CIHR-INMHA also created a Chair in Neuroethics whose first titular will be announced in spring 2007. Support by CIHR-INMHA is critical in this highly innovative area since other sources of funding are scarce. CIHR-INMHA is also leading an international group of research funders from Asia, Europe and North America. We plan to provide funding to expert teams in this area as CIHR-INMHA and Canada are now seen as the world leaders in Neuroethics (ibid.).

The RFAs (i.e., grant proposal solicitations) that are referred to in the statement above were both awarded to Dalhousie University, to two different teams of researchers [35]. Each grant was for $\$ 1.5$ million (CAD) for a five-year period. With the addition of the Chair in neuroethics, CIHR-INMHA will have allocated over $\$ 4$ million to supporting research in neuroethics. While this may not seem like a lot of money, at least compared to funding for ELSI issues in nanotechnology or in genetics, this sum represents one of the largest governmental investments in neuroethics in the world (inferred from chart on p. 981 in [20]). One other recent funding initiative is also worth mentioning. In late April 2007, a call for proposals for neuroethics projects was issued through a joint effort from Germany, Canada, and Finland. Funding is for approximately $\$ 1.3$ million (USD), over a three year period from the Canadian and Finnish funding agencies [5, 44].

While the existence of multi-millions of dollars of federal funding (and some private foundation funding, such as from the Dana Foundation) does not provide proof in and of itself of the increased research activity in ELSI issues in genetics and in nanotechnology, it is clear 
that researchers have been applying for and receiving funding in these areas for several years. Moreover, in both the case of the Human Genome Project and the National Nanotechnology Initiative, Congress (either at the outset or shortly after the beginning of each project) mandated that a part of the federal budgets for each program be set aside for studying the ethical, legal, and social implications of the respective projects (see e.g., [8], p. 335; [37], Sec. 2(b)(10)), meaning that both the overarching projects and the study of ELSI issues were seen as national priorities, with a relatively steady funding stream. Again, in the US, this has not been the case with the study of various aspects of the neurosciences, including neuroethics.

\section{Who is Addressing the Issues?}

There is another factor that encourages separate ethical inquiries into nanotechnology and neurotechnology; academics and other researchers engaging the ethical issues seem to be coming from at least two different perspectives on ethics. Those who pursue nanoethics tend to come from backgrounds in engineering, hard sciences, Science and Technology Studies, and/or the social sciences (political science, sociology, anthropology, economics), while those who pursue neuroethics tend to come more from the humanities, the neurosciences, and medicine.

To find evidence of this, I performed a search on grants awarded by the National Science Foundation ${ }^{6}$ for ELoSI issues in nanotechnology. I focused the search on grants awarded specifically from the Directorate for Social, Behavioral, and Economic Sciences, since most ELoSI type grants would be funded through that directorate. The search string for awards I used was "ethics OR ethical OR social OR societal OR society", and the program search term was "nano*". This search resulted in 22 grants between July 2002 and September 2006 and totaled approximately $\$ 15$ million. There are 21 separate Principal Investigators (PIs) represented in these 22 grants. Of these $21 \mathrm{PIs}$, fully 19 came from backgrounds in engineering, science and technology studies, chemistry, physics, economics, anthropology,

\footnotetext{
${ }^{6}$ Search engine located at http://www.nsf.gov/awardsearch/.
}

or sociology. The remaining two PIs were philosophers who focused on either philosophy of chemistry or on philosophy and agricultural economics. The two remaining PIs of the original 21 were the only ones who had published extensively in the bioethics realm. They are both academic attorneys.

I then further narrowed the original search parameters to omit "social, society, and societal" as search terms. Searching for only "ethics OR ethical" elicited 9 grants (a subset of the original 22 grants), totaling approximately $\$ 4.4$ million. All of these grants were awarded to either social scientists, engineers, or philosophers who focus on chemistry or agricultural economics. These statistics are understandable given that it is scholars from the above types of disciplines who would be more likely to apply for and receive grants from the National Science Foundation, since NSF is the funding agency in the federal government that predominantly provides grant opportunities in these fields, whether related to nanotechnology or not.

There is less of a track record to examine in neuroethics, since there have been only two major grants awarded, in Canada, explicitly for neuroethics. However, both these grants were awarded to PIs whose primary work has been in bioethics and health policy. In the US, any grant solicitations that could conceivably include discussions or examinations of ELSI issues in the neurosciences are granted from the National Institutes of Health. The scholars and scientists who tend to apply for these grants are much more oriented toward biomedical fields (as are NIH's institutes and centers). That said, NIH is part of the National Nanotechnology Initiative, although its focus is particularly on research that relates to nanotechnology applications in medicine. It is mostly in this context that a very small handful of bioethics researchers have delved into any issues related to nanotechnology. This is not especially surprising, given that most of the applications of nanotechnology go beyond the usual scope of issues that those in bioethics normally address (e.g., dealing with the properties of materials).

So, it seems that as research into "nanoethics" and "neuroethics" has proceeded, "never the twain shall meet." What is the problem with this state of affairs? It may mean that the result of ethical inquiries into each area will not easily transfer or be shared across disciplinary boundaries. The scholars addressing each set of ethical issues may publish in broadly philosophically- 
oriented journals (as an example), but they are much more likely to publish articles in journals geared to either nanotechnology OR neuroscience/bioethics/ health policy. In addition, there are new journals for each sub-field of ethics. For instance, Springer Netherlands recently started a journal called Nanoethics and has just started the journal in which this article appears, Neuroethics. In the neuroethics field, there is also The American Journal of Bioethics Neuroscience which began publishing in early 2007. So, now it seems that the venues for publication in these fields may well reinforce that separation. Given this prospect, it is appropriate to next examine the types of ethical issues that may arise in nanotechnology and neuroscience, to see what the potential consequences of separation might be.

\section{What are the Ethical Issues and How Do They Arise?}

First, how different are the ethical issues raised by nanotechnology and neuroscience (the two of interest here)? Second, is it beneficial for inquiries into these issues to proceed on different trajectories (which seems to be the case now)?

At the most basic level, there are two categories of concerns that arise from nearly all scientific and technological endeavors: (1) those relating to more technological aspects of engineering (i.e., what technologies enable us to do), and (2) those based more on philosophical questions about the human condition and about the aims of science.

These two types of questions are, in many ways, inseparable - and I do not intend to imply otherwise. However, I have separated them to illustrate that ethical questions can arise from broad questions about the human species, and from specific questions about the implications of a particular technological application. This distinction will be important to bear in mind as we directly address how to overcome the quandary of myopic or subdivided ethics.

Some examples of very broad questions that one might ask under the category of "what technologies enable" could be:

1. Does the science/technology application pose physical or environmental harms? If so, can we mitigate them and how?
2. Does the science/technology application pose potential physical or psychological harms to people? If so, can we mitigate them? If so, how?

3. Are there potential collateral uses (beneficial and harmful) of the science/technology that need to be recognized and addressed up front?

4. How might a particular neural device implanted in a patient's brain effect their cortical functioning? Assuming the device is found to be sufficiently safe to implant, how do we design an informed consent process that identifies the risks involved?

Among the broad philosophical questions that can be asked, we have:

1. What is the aim of the science/technology research/application?

- How will the science/technology being developed/ applied address that aim?

- Are the resources being spent on the specific aim the best or most appropriate use of those resources?

2. What is our understanding of what it means to be human?

- How do we view the "proper" role of science and technology in shaping or defining who and what we are as a species? As a society? As individuals?

- What does it mean for our "humanness" if we incorporate technologies into our bodies?

3. What impact will the technologies have on our relationship to our environment (broadly construed)?

4. How will new technologies affect society's "haves" vs "have nots" (will the divide become larger or different in significant ways?) (i.e., distributive justice issues).

Let us now move to discuss the more specific ethical issues that have been or are being addressed within nanotechnology and neuroscience. There are actually many issues that are being addressed by, or are relevant to, both nanotechnology and neuroscience applications, and in similar contexts. For instance, human enhancement is an issue that is of concern in both realms. In discussions of nanotechnology (and specifically, nanomedicine), longevity is a major issue - nanotechnologies could be applied to human cells (including bone cells) to either prevent them from aging, breaking down or otherwise to augment the cellular structure. In the 
neurosciences, human enhancement discussions revolve around cognitive enhancement (e.g., through neural prostheses, or, more commonly at present, through psychopharmaceuticals) or sensory enhancement. In both nano and neuro contexts, enhancement implies augmentation of existing (and likely properly functioning) capacities, versus restoring abilities that may have been lost. For the moment, less controversy surrounds the use of technologies to restore lost abilities.

A second common theme in nanotechnology applications and neuroscience advances concerns surveillance and privacy. In the case of nanotechnology, the possibility of microscopic sensors that can read/transmit data about people (their movements, their bodily functions, their location, etc.) is a major area of concern. For neuroscience applications, concerns are particularly acute in discussions of functional neuroimaging and the application/misapplication of information inferred from the images. Among the most controversial potential applications is the use of functional neuroimaging to "detect" when someone is lying. Indeed, there are already two private companies in the $\mathrm{US}^{7}$ that are selling their functional neuroimaging technologies and access to proprietary software for lie detection purposes. What makes these applications controversial is that images from functional Magnetic Resonance Imaging (fMRI; upon which the lie detection applications are based) depicts "metabolic correlates of neural activity, not the activity itself. Images are constructed based on blood-oxygenation-level dependent (BOLD) contrast" ([21], p. 156). Most troubling, however, is the fact that there is little reason to think that the neural activity itself (given our current understanding - or lack thereof) can be reliably interpreted as indicative of deception. So, conclusions are being drawn on the basis of interpreting blood oxygenation levels in the brain (which are not necessarily consistent from person to person) and taken to mean that a person is lying $-\mathrm{a}$ potentially spurious conclusion. Moreover, the fact that there are now commercial applications for these and some nanotechnology applications raises other common issues of safety, risk, and regulation. Some of these questions will be ethical in nature while others will be matters for legal and public policy processes.

A third common ethical issue raised in nanotechnology and neuroscience applications concerns the

\footnotetext{
${ }^{7}$ Cephos Corporation, based in Pepperell, MA; and No Lie MRI. In Phoenix, AZ.
}

use of these technologies for military applications. The US military has been conducting and funding much research in the neurosciences, particularly for cognitive and sensory enhancement research. Some of the controversies arising in this context concern applications that may enhance a warfighter's (the term used by the Defense Advanced Research Projects Agency [DAPRA] for "soldier") ability to kill, or to minimize the psychological impact of having done so. To the extent that nanotechnologies will play a role in these applications, they will be implicated as well.

Possibilities of other types of human harm from applications of nanotechnologies and of neurotechnologies will also raise ethical issues. Among the issues of concern in nanotechnologies is the probability that they will be able to traverse the blood-brain barrier. While for many applications (e.g., brain tumor treatment), this characteristic is desirable, it may also be that in other applications, it is clearly not only undesirable, but also fraught with risks for proper cognitive function. Inhaling or ingesting nanostructures (e.g., carbon nanotubes) may cause harms as they interact with bodily tissues. For technologies associated with neuroscience, some of the issues of possible harm arise from implantable neural devices (such as brain-computer interface chips), "smart drugs" (non-medical uses of psychopharmaceuticals), or inappropriate uses of functional neuroimages. Many of the harm issues (for both nano and neuro) will also be byproducts of the other ethical issues these technology applications raise, e.g., potential harms associated with the use of nanotechnologies for surveillance purposes.

Finally, for this discussion at least, there is the perennial issue that afflicts most discussions of ethics and technologies: that the ethics considerations lag behind the technology developments, or that the ethical discussions that do occur do so in a vacuum. Some have argued that this is what happened with the Human Genome Program's ELSI research (see, e.g., $[4,12,23,15])$, i.e., that ELSI researchers conducted their work in isolation from either the policy process and/or genomic scientists. That criticism was not lost on Congress; the legislative language mandating the contemplation of ELSI issues in nanotechnology set out the following stipulation:

insofar as possible, integrat[e] research on societal, ethical, and environmental concerns with nanotechnology research and development, and 
ensur[e] that advances in nanotechnology bring about improvements in quality of life for all Americans [P.L.108-153 2003, Sec. 2(b)(10)(C)].

The extent to which this mandate allows, encourages, or even rewards relevant ethical considerations beyond those strictly related to nanotechnology is not clear. Certainly to date, the major US federal government entities funding nanotechnology initiatives have not seemed to encourage such extra-disciplinary collaboration. For instance, a recently awarded NSF grant for "nanoethics" provided $\$ 250,000$ for a three year project to study the ethics of human enhancement [36]. The project's description acknowledges that there is an existing literature on human enhancement, but it also makes clear that the investigators consider nanotechnology to be the driving force for their study. Interestingly, in a press release about the grant award, the following were among the questions the investigators stated they will consider:

What exactly constitutes enhancement? Is there a right to be enhanced? Is it justifiable to enhance people in order for them to undertake certain tasks, e.g., in the military? Is there an obligation to enhance our children? Should there be limits on the types of enhancement allowed or the degree to which someone can be enhanced? Does it make an ethical difference if some enhancing device is implanted into the body rather than worn on the outside? Does the notion of human dignity suffer with such enhancements? [29]

In this list of questions, there is little to suggest why they solely relate to nanotechnology. Indeed, as mentioned above, questions of human enhancement have been considered from a philosophical perspective (within specific contexts, but also from a more general perspective) for many years. Moreover, it must be remembered that "nanotechnology" refers to the SIZE of the technology, and not to a specific site or mode of application.

The intent here is not to single out any particular grant for criticism - it is merely to indicate that the tendency to subdivide ethical inquiries into specialized areas of discourse may inevitably lead to multiple, parallel, and separate endeavors, impeding possible cross-fertilization of ideas, and leading to the real possibility that the fundamental underlying philosophical issues will be viewed very differently, depending upon the domain of discourse. Using the example of human enhancement above, it may be that by separately contemplating the fundamental questions specifically relevant to nanotechnologies, enhancements will be seen as acceptable, whereas in the context of neurotechnologies, they will not. In this case, it may get even more confusing as nanotechnologies find more and more applications in the "neuro" context. Indeed, it will be the case that nanotechnologies are going to be applied in many neuro-related applications; for instance, one application in the conceptual stage would use nano-electrodes (which can read and deliver electrical signals) threaded through an artery in the groin up to the brain. These electrodes could then be used to deliver deep brain stimulation (DBS) for patients with Parkinson's Disease. Because the electrodes are only $600 \mathrm{~nm}$ wide, they can get much closer to the effected neurons than can conventional electrodes used in DBS $[25,7]$.

It is also the case that those researching issues in neuroethics are, by and large, not focusing on nanotechnologies or on nanoethics. Certainly there has been some acknowledgement that nanotechnologies will have an impact on clinical neurosciences at some point in the future. There have been some articles published that, like this article, question the wisdom of subdivided ethical inquiries [46, 38]. Other articles question whether there is anything "new" in either domain that warrants such separate inquiries [24, 39]. In any case, I would argue that for those in the neurosciences or studying "neuroethics" specifically, it is folly to not consider the role nanotechnologies will play in clinical neurosciences. This is especially true given the evidence that many technologies are beginning to converge, and will continue to do so, and at a more rapid pace, in the future. Indeed, the NSF has already coined an acronym for these converged technologies - NBIC (or Nano-Bio-InfoCogno; see, e.g., [2, 40]. Interestingly, many of the applications of nanotechnology which generate a lot of interest in the nanotechnology literature are those whose primary application is within the brain, central nervous system, or peripheral nervous system.

And, lest anyone think that the issues identified above are only common to nanoethics and neuroethics 
inquiries, they should be assured that each of these issues has already been raised in the context of the genetic sciences. To a large extent, many of these common issues have been the subject of HGP ELSI funded research. Human enhancement and identity, privacy, access and distributive justice issues, and the lag between ethics and technology - all these issues have been addressed on either the philosophical and/or contextual levels for the genetic sciences and technologies. The question is whether any of the fruits of that labor will be utilized as similar questions arise in nanotechnology and neuroscience applications. Given that many of the bioethics researchers who have addressed ethical issues in the context of genetics are the same ones who are now undertaking ethical inquiries into neurosciences and neuro-technologies, I am more optimistic as to the prospects of the earlier work being applied in the neuroethics domain. As stated earlier, it does not appear as yet that the researchers who are examining nanotechnologies' ethical implications are doing the same, although additional research is needed to test the validity of this hypothesis.

\section{Difference of Degree or Difference of Kind? (or, is There Anything New Here?)}

It is necessary at this juncture to squarely address the question of whether there is any value in subdividing or contextualizing domains of ethical inquiry. Others, as mentioned above, have addressed this question by asking whether there is anything new arising from the technologies to warrant the separation. I will address this question by asking whether the ethical issues arising in the nano and neuro contexts represent a "difference of degree" or a "difference of kind." Again, I turn to the distinction I made earlier about the ways in which ethical issues arise in scientific and technological endeavors (either through what technologies enable, or through the more fundamental philosophical questions about the nature of man and the aims of science). I contend that the answer to the question of degree or kind depends on the category of the question. For the practical (i.e., technologyenabling) questions, there could be a point in time at which there will be "differences in kind" - i.e., that the capabilities that these technologies might enable could be so radical as to constitute a different species of question/issue. For the fundamental philosophically based ethics questions, however, there will likely only be a difference of degree; that is, the underlying issues will still revolve around questions about the nature of man, distributive justice, or the aims of science. That said, as stated at the outset, it may not be entirely obvious in all cases just how to characterize the nature of the issues, or at what point a difference of degree becomes a difference of kind. Clearly, determining the "starting point" will be crucial in making that determination. Consider the following illustration of human enhancement:

These three photographs show different states of being (although, admittedly, photo 3 is not a direct progression from the two others - i.e., the third is of a different fictional character). Philosophically, the questions that underlie the state of being portrayed in each photo, and the progression from one to the

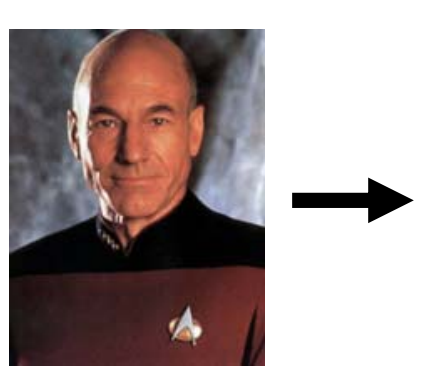

1

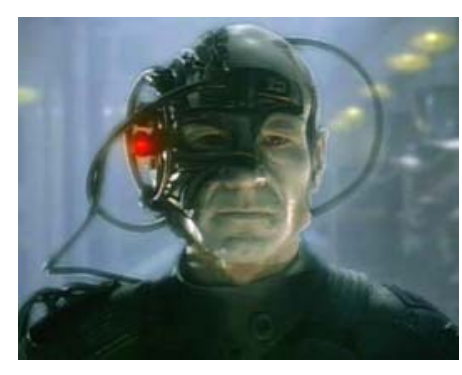

2

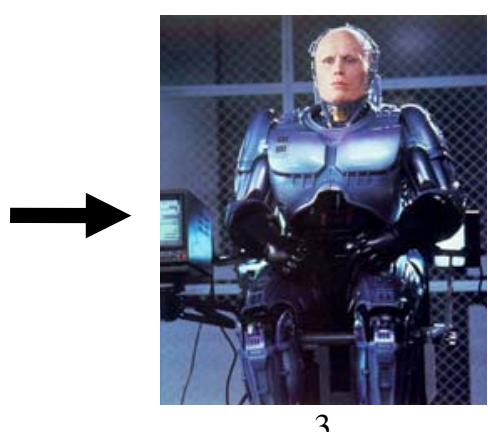

3

"Star Trek: The Next Generation" - photos courtesy of CBS Paramount Network Television. RoboCop photo courtesy of the RoboCop Archive. 
next, are identical: what is the nature of man; what do we mean by the concept of "human"? It does not matter whether we start at photo 1 and jump to photo 3 - the questions are the same, and at most, represent a difference of degree (e.g., "Is Robocop any less human than Jean Luc Picard?"). If we look at the photos from the perspective of the application of technology, however, the question of whether something represents a difference of degree or of kind is less clear. For instance, if we start at photo 1 and jump to photo 3 , the progression seems to represent a difference in kind. However, if we start at 1 and go to 2 or start at 2 and go to 3 , both these seem to represent a difference of degree. Again, the starting point makes the difference. ${ }^{8}$ The appropriateness of the use of the technology for these specific applications is among the ethical questions arising from these photos. It is also interesting to note that going sequentially from photo 1 to 3 may elicit fewer ethical questions (on the face of it, at any rate), than the jump from photo 1 to photo 3 . In other words, the more incremental the technological changes seem to be, the fewer ethical issues will likely be raised along the way. This does not mean, however, that this is necessarily the appropriate lens through which to view these changes.

So, bringing this back to the current discussion emphasizing the point that ethicists addressing neuroapplications need to be looking at nanotechnologies - the increasing integration of nanotechnologies into neuroapplications will likely yield issues differing in degree (partly due to the "starting point" issue). Examples of the issues that would be relevant include,

- The adequacy of how we address risk;

- The protection of human subjects in research;

- The regulation of medical devices;

- Social benefits and harms of the emerging technologies;

- The impact of the technologies on the practice of clinical medicine; and

- The additional expertise required of medical personnel to use nano-enabled technologies.

\footnotetext{
${ }^{8}$ Another example might be the progression of the biplane to the space shuttle, as examples of transportation technologies. Viewed as endpoints, the technologies of the biplane and the shuttle represent a difference of kind. Viewed incrementally, the technologies may represent merely a difference of degree from one to the next.
}

However, there may also come a point at which the outcome resulting from these incrementally addressed issues will be something so different from what was originally contemplated as to become an utterly new species of issue.

\section{Lessons to be Learned}

There is no question that the relevant technological artifacts are becoming increasingly sophisticated, that they are shrinking in size while increasing in capabilities, and are converging across modalities (e.g., NBIC technologies). Since nanotechnologies could be applied to many different scientific and clinical endeavors, there is also little question that nanotechnologies will have a greater impact on clinical neuroscience than neuroscience will have on nanotechnology. As such, two things become clear: as mentioned above, those focusing on neuroethics cannot afford to ignore the impact (technologically and ethically) nanotechnology will have on neuroscience and brain function; and those focusing more on nanoethics should heed the underlying philosophical debates that have been occurring for decades in other domains of ethical inquiry. Otherwise, we risk constantly "reinventing the same wheel" over and over again, with little opportunity to learn from the rich and diverse literature produced from past inquiries into similar issues/questions. Not only is this a waste of intellectual capital, it is also likely a waste of the taxpayer dollars being spent on grants pursuing what may well be duplicative ethical inquiries.

We need to resist the temptation to categorize all ethical questions by domains of inquiry. Bioethicists claim to reject reductionist perspectives, and yet have been caught up in it in the genetic and now, neuro contexts. It appears that researchers addressing ethical issues in nanotechnologies are doing the same. (Again, more research is needed to address this supposition.) By focusing on reductionist or alternatively, myopic approaches to ethical implications of technologies, we miss the opportunity to learn from other contexts in which the issues have been addressed, already taking into account the more deeply rooted philosophical questions. Indeed there is already convergence in the issues now - a trend that will intensify into the future. 
I would contend that researchers focused on either nanoethics or neuroethics (or other subdivided ethics) should resist the temptation that the funding agencies perpetuate - i.e. to look myopically at all of the ethics issues within their own particular context, and instead focus on collaborative opportunities for addressing at least the underlying philosophical questions that are relevant to ALL scientific/technological endeavors. Of course, this also means that funding agencies should provide funding mechanisms that encourage (or indeed, mandate) these sorts of collaborative opportunities across contextual settings where otherwise there would be no natural collaborations. By this discussion, I do not intend to imply that there is no place for contemplating any ethical issues through the domain of technology application. For the ethical questions of a technological nature, an understanding of the specific technologies and applications will be invaluable.

I end with what I call the elephant metaphor of myopic ethics. There is an old Indian (or Jain) parable in which six blind men come across an elephant. None had ever "experienced" an elephant before. They moved close to it so they can feel it and describe what they felt, each at a different part of the elephant's body. The man by the elephant's leg described it as a pillar, the man near the ear described it as a fan, the torso was described as a wall, the tail as a rope, etc. Each man experienced only a part of the elephant, but each insisted he was correct in describing the whole elephant. The problem, of course, was that none of the blind men could "see" the entire elephant, and thus could not comprehend what the elephant really was.

We run the same sort of risk with the overlapping ethical issues that arise in the contexts of nanotechnology and the neurosciences/neurotechnologies, and other scientific and technological domains in which ethical issues arise. There is much to gain from the expertise and insights ethics researchers have acquired in their own domains of inquiry. We now need to be wise enough to learn how to share this intellectual wealth, and encourage researchers to do so.

Acknowledgement The author is grateful to Vaughn McKim, Andrew Fenton, and Neil Levy for their insightful comments on earlier drafts of this article. Support for this research was provided by a grant from the Canadian Institutes for Health Research.

\section{References}

1. Abe, N., M. Suzuki, E. Mori, M. Itoh, T. Fujii. 2007. Deceiving others: Distinct neural responses of the prefrontal cortex and amygdala in simple fabrication and deception with social interactions. Journal of Cognitive Neuroscience 19(2): 287-295.

2. Bainbridge, W., M. Roco. 2006. Managing nano-bio-infocogno innovations: Converging technologies in society. The Netherlands: Springer.

3. Baughman, R., R. Farkas, M. Guzman, M. Huerta. 2006. The National Institutes of Health blueprint for neuroscience research. The Journal of Neuroscience 26(41): 10329-10331.

4. Bennett, I., D. Sarewitz. 2006. Too little, too late? Research policies on the societal implications of nanotechnology in the United States. Science as Culture 15(4): 309-325.

5. BMBF. 2007. Neuroethics initiative. Retrieved July 20, 2007 from http://www.gesundheitsforschung-bmbf.de/ en/1592.php. German Federal Ministry of Education and Research (Bundesministeriums für Bildung und Forschung).

6. Boyer, J. 2007. ELSI funding. S. Alpert, personal communication. July 13, 2007.

7. Bullis, K. 2006. Tiny electrodes for the brain. Technology Review. Retrieved February 6, 2007 from http://www. technologyreview.com/printer_friendly_article.aspx? $\mathrm{id}=16755$.

8. Burris, J., R. Cook-Deegan, B. Alberts. 1998. The Human Genome Project after a decade: Policy issues. Nature Genetics 20: 333-335.

9. Chambers, E., S. Mitragotri. 2007. Long circulating nanoparticles via adhesion on red blood cells: Mechanism and extended circulation. Experimental Biology and Medicine 232(7): 958-966.

10. Chen, X., A. Kis, A. Zettl, C. Bertozzi. 2007. A cell nanoinjector based on carbon nanotubes. Proceedings of the National Academy of Sciences 104(20): 8218-8222.

11. Decety, J., P. Jackson, J. Sommerville, T. Chaminade, A. Meltzoff. 2004. The neural bases of cooperation and competition: An fMRI investigation. NeuroImage 23: 744-751.

12. Editorial. 2001. Defining a new bioethic. Nature Genetics 28(4 (August): 297-298.

13. Fehr, E., U. Fischbacher, M. Kosfeld. 2005. Neuroeconomic foundations of trust and social preferences: Initial evidence. American Economic Review 95(2): 346-351.

14. Feynman, R. (1959). There's plenty of room at the bottom. Retrieved July 2, 2007 from http://www.zyvex.com/nano tech/feynman.html.

15. Fisher, E. 2005. Lessons learned from the Ethical, Legal and Social Implications Program (ELSI): Planning societal implications research for the National Nanotechnology Program. Technology in Society 27: 321-328.

16. Gazzaniga, M. 2005. The ethical brain. New York: Dana.

17. Genomics.energy.gov. 2004. Human Genome Project budget. Retrieved June 28, 2007 from http://www.ornl.gov/sci/ techresources/Human_Genome/project/budget.shtml.

18. Glannon, W. 2007. Bioethics and the brain. Oxford: Oxford University Press.

19. Illes, J. 2003. Neuroethics in a new era of neuroimaging. American Journal of Neuroradiology 24: 1739-1741. 
20. Illes, J., C. Blakimore, M. Hansson, Tl. Hensch, Al. Leshner, G. Maestre, P. Magistretti, R. Quirion, P. Strata. 2005. International perspectives on engaging the public in neuroethics. Nature Reviews Neuroscience 6(December): 977-982.

21. Illes, J., E. Racine, M. Kirschen. 2006. A picture is worth 1000 works, but which 1000? Neuroethics: Devining the issues in theory, practice, and policy. J. Illes. Oxford: Oxford University Press.

22. INMHA. 2007. Institute of Neurosciences, Mental Health and Addiction strategic plan 2007. Retrieved July 16, 2007 from http://www.irsc.gc.ca/e/documents/inmha_strategic_ plan_2007_e.pdf.

23. Kitcher, P. 2001. Science, truth, and democracy. Oxford: Oxford University Press.

24. Litton, P. 2007. Nanoethics? What's new? Hastings Center Report 37(1): 22-25.

25. Llinas, R., K. Walton, M. Nakao, I. Hunter, P. Anquetil. 2005. Neuro-vascular central nervous recording/stimulating system: Using nanotechnology probes. Journal of Nanoparticle Research 7: 111-127.

26. Maynard, A. 2007. Nanotechnology: Benefits and risks (testimony before the President's Council on Biomedical Ethics). Retrieved July 5, 2007 from http://bioethics.gov/ transcripts/june07/session4.html, June 29.

27. McClure, S., et al. 2004. Neural correlates of behavioral preference for culturally familiar drinks. Neuron 44: 379-387.

28. Nanocompositech. 2007. Glossary (nanoscience definition). Retrieved June 25, 2007 from http://www.nanocomposi tech.com/glossary-nanocomposite-nanotechnology.htm.

29. Nanoethics.org. 2006. Nanoethics researchers awarded $\$ 250,000$ from US National Science Foundation. Retrieved August 12, 2007 from http://www.nanoethics.org/ rls092506.html.

30. Nanotech-Project. 2007. Analysis. October 2. Project on Emerging Nanotechnologies of the Woodrow Wilson International Center for Scholars. Retrieved January 22, 2008 from http:/www.nanotechproject.org/inventories/consumer/ analysis.

31. NHGRI. 2004. NHGRI launches centers for excellence in ethical, legal and social implications research. Retrieved July 11, 2007 from http://www.genome.gov/12512375.

32. NHGRI. 2007. The ELSI Research Program abstracts and activities database. Retrieved July 23, 2007 from http://genome. gov/page.cfm?pageID=17515632. (search term "ethical").

33. NNI. 2006. The National Nanotechnology Initiative: Research and development leading to a revolution in technology and industry. Supplement to the President's FY 2007 budget, July 2006. Retrieved June 28, 2007 from http://www.nano.gov/NNI_07Budget.pdf.

34. NNI. 2007. National Nanotechnology Initiative: FY 2008 budget and highlights. Retrieved June 26, 2007, from http://www.nano.gov/pdf/NNI_FY08_budget_summaryhighlights.pdf.

35. noveltechethics.ca. 2007. Neuro Research Projects. Retrieved July 20, 2007 from http://www.noveltechethics.ca/ site_neuro_project.php?page $=9$.

36. NSF. 2006. Collaborative research: Nanotechnology and human enhancement (Award 0621021). Retrieved August 12, 2007 from http://www.nsf.gov/awardsearch/show Award.do?AwardNumber $=0621021$.

37. P.L.108-153. 2003. 21st Century Nanotechnology Research and Development Act. 15 USC 7501.

38. Parens, E., Johnston, J. 2006. Against hyphenated ethics. Bioethics Forum. Retrieved September 6, 2006 from http:// www.bioethicsforum.org/genethics-neuroethics-nanoethics.asp.

39. Parens, E., J. Johnston. 2007. Does it make sense to speak of neuroethics? EMBO Reports 8(Special Issue): S61-S64.

40. Roco, M., Bainbridge, W., ed. 2002. Converging technologies for improving human performance: Nanotechnology, biotechnology, information technology and cognitive science. Arlington, Virginia, National Science Foundation/ Department of Commerce.

41. Savage, L. 2007. Press release: Nanoparticles carry chemotherapy drug deeper into solid tumors. Journal of the National Cancer Institute 99(13): 981-c-.

42. Schmidt, K. (2007). Nanofrontiers: Visions for the future of nanotechnology. Washington, DC, Project on Emerging Nanotechnooogies. Retrieved from http://www.nanotech project.org/reports.

43. Tankersley, D., C.J. Stowe, S. Huettel. 2007. Altruism is associated with an increased neural response to agency. Nature Neuroscience 10: 150-151.

44. Tirronen, M. 2007. Question regarding joint neuroethics research projects. S. Alpert, personal communication, August 18, Helsinki.

45. Tovino, S. 2007. Functional neuroimaging and the law: Trends and directions for future scholarship. American Journal of Bioethics - Neuroscience 7(10).

46. Wilfond, B., V. Ravitsky. 2005. On the proliferation of bioethics sub-disciplines: Do we really need "genethics" and "neuroethics"? American Journal of Bioethics 5(2): 20-21. 